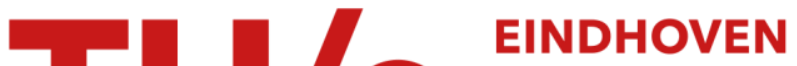

\section{Graphic method to determine the angular acceleration of links of compound mechanisms}

\section{Citation for published version (APA):}

Dijksman, E. A. (1966). Graphic method to determine the angular acceleration of links of compound mechanisms. Journal of Mechanisms, 1(1), 73-81. https://doi.org/10.1016/0022-2569(66)90008-5

DOI:

10.1016/0022-2569(66)90008-5

Document status and date:

Published: 01/01/1966

\section{Document Version:}

Publisher's PDF, also known as Version of Record (includes final page, issue and volume numbers)

\section{Please check the document version of this publication:}

- A submitted manuscript is the version of the article upon submission and before peer-review. There can be important differences between the submitted version and the official published version of record. People interested in the research are advised to contact the author for the final version of the publication, or visit the $\mathrm{DOI}$ to the publisher's website.

- The final author version and the galley proof are versions of the publication after peer review.

- The final published version features the final layout of the paper including the volume, issue and page numbers.

Link to publication

\section{General rights}

Copyright and moral rights for the publications made accessible in the public portal are retained by the authors and/or other copyright owners and it is a condition of accessing publications that users recognise and abide by the legal requirements associated with these rights.

- Users may download and print one copy of any publication from the public portal for the purpose of private study or research.

- You may not further distribute the material or use it for any profit-making activity or commercial gain

- You may freely distribute the URL identifying the publication in the public portal.

If the publication is distributed under the terms of Article 25fa of the Dutch Copyright Act, indicated by the "Taverne" license above, please follow below link for the End User Agreement:

www.tue.nl/taverne

Take down policy

If you believe that this document breaches copyright please contact us at:

openaccess@tue.nl

providing details and we will investigate your claim. 
J. Mechanisms Vol. 1, pp. 73-81. Pergamon Press 1966. Printed in Great Britain

\title{
GRAPHIC METHOD TO DETERMINE THE ANGULAR ACCELERATION OF LINKS OF COMPOUND MECHANISMS
}

\author{
Dr. E. A. DUKSMAN \\ Technological University Eindhoven, The Netherlands
}

(Received 10 June 1965)

\begin{abstract}
For a mechanism of bars, the links being connected by turning pairs, a graphic method has been developed to determine the angular acceleration of each link of the mechanism. This is done by splitting up each $n$-side polygon of the mechanism into $(n-3)$ four-bar linkages, each of which contributes to the angular acceleration of one side of the polygon with respect to the next one in turn. An additional contribution represented by a mixed term has to be taken into account.

In this way we find for each polygon two independent relations between the angular accelerations of the sides. In connection with the relations of the kind $\dot{\omega}_{i j}+\dot{\omega}_{j k}=\dot{\omega}_{i k}$ the method makes it possible to calculate the angular acceleration of each link of the mechanism.
\end{abstract}

\section{INTRODUCTION}

WrTH many compound mechanisms it is not very easy to determine the angular acceleration of each link. Rosenauer [1] made use of the plan of relative normal accelerations, which was introduced by him. With the aid of this plan he always could solve the problem. However, as the acceleration vectors in this plan always have to form a closed diagram, it is imaginable that with certain compound mechanisms a deep geometrical understanding is required to find the solution. Moreover the method of Rosenauer has to be repeated to determine the complete course of accelerations of a certain point of the mechanism. This makes the method impracticable in several cases.

For this reason a method has been developed which only demands the measurement of linesegments to determine the angular acceleration of a link of the mechanism. The solution, presented in this paper, can be applied to plane mechanisms with one degree of freedom, the links being connected by joints.

We imagine such a mechanism being composed of a certain number of polygons, which are connected to one another in such a way, that there is only one degree of freedom to be left. Besides this, different polygons often have a link in common (this is taken into account by the way of numbering the links of the mechanism). For each polygon of $n$ sides two independent equations can now be formed for the angular velocities, as well as for the angular accelerations. This is true because the angular position of $(n-3)$ links of each free polygon relative to a $n$th link, which is considered to be fixed, can be chosen freely. So only two links are left, the motion of which relative to this nth link is fixed.

It will be clear, the mechanism having one degree of freedom, there can be indicated enough polygons to determine all angular accelerations of the links. Besides, the number of the necessary equations can be reduced to a minimum by right choice of the polygons and of the fixed link in each of them. 


\section{THE POLYGON}

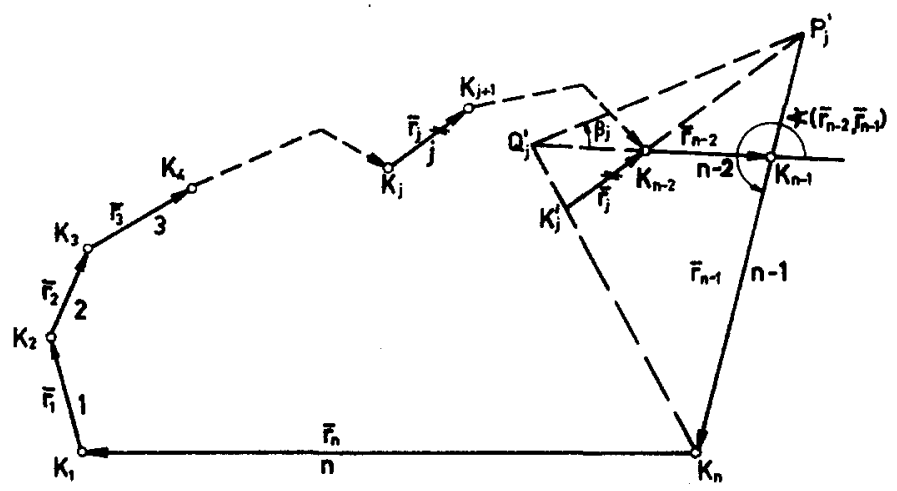

FiG. 1. $n$-side polygon.

2.1 The equations of the angular velocities in the polygon

In Fig. 1 the numbers of the sides follow each other clockwise, the fixed side receiving number $n$. Do we see the polygon as a closed vector-polygon, we can write

$$
\bar{r}_{1}+\bar{r}_{2}+\ldots+\bar{r}_{n}=\overline{0}
$$

We agree upon the joint, indicated by $K_{j}$, being the starting point of the vector $\bar{r}_{j}$. On the ground of an equation, derived by Euler, we have, between the vectors of velocity of the joints $K_{j+1}$ and $K_{j}$, the relation

$$
\bar{v}_{K_{j+1}}=\vec{v}_{K_{j}}+\left(\bar{\omega}_{j, n} \times \bar{r}_{j}\right)
$$

$\bar{\omega}_{j, n}$ being the vector of angular velocity of side $j$ relative to side $n$. Summation on $j$ yields

$$
\bar{v}_{K_{n-2}}=\sum_{j=1}^{j=n-3}\left(\bar{\omega}_{j, n} \times \bar{r}_{j}\right)
$$

Each vector product of this sum contributes to the resulting velocity of joint $K_{n-2}$ and consequently to that of joint $K_{n-1}$. It is possible to calculate separately the contribution of each vector product to the angular velocity of the $(n-1)$ th link with respect to the $n$th link, and to do the adding afterwards. For, each contribution can be determined separately with the help of a closed quadrilateral. This quadrilateral can be formed by a vector $\bar{r}_{j}$, moved parallel to itself and terminated in pivot point $K_{n-2}$, the vectors $\bar{r}_{n-2}$ and $\bar{r}_{n-1}$ and the closing vector. The angular velocity,$\omega_{n-1, n}$ of link $n-1$ with respect to link $n$ owing to the angular velocity $\omega_{j, n}$ of the shifted vector $\bar{r}_{j}$, is determined in the four-bar linkage by measuring the transmission ratio $i_{n-1}, j$. We indicate the displaced starting point of $\bar{r}_{j}$ by $K_{j}^{\prime}$ and the intersection of the vectors $\bar{r}_{n-2}$ and $\overrightarrow{K_{n} K_{j}^{\prime}}$ by $Q_{j}^{\prime}$. Hence 


$$
\frac{\omega_{n-1, n}}{\omega_{j, n}}=i_{n-1, j}=\frac{\overline{Q_{j}^{\prime} K_{j}^{\prime}}}{\overline{Q_{j}^{\prime} K_{n}}}
$$

a ratio which can be indicated directly in the diagram. Summation on $j$ yields

$$
\omega_{n-1, n}=\sum_{j=1}^{j=n-3} \omega_{n-1, n}=\sum_{j=1}^{j=n-3} i_{n-1, j} \cdot \omega_{j, n}=\sum_{j=1}^{j=n-3} \overline{\overline{Q_{j}^{\prime K} j}} \omega_{j, n}
$$

This is the first equation between the angular velocities of the polygon. In the same way we have for the angular velocity of link $n-2$

$$
\omega_{n-2, n}=\sum_{j=1}^{j=n-3} \omega_{n-2, n}=\sum_{j=1}^{j=n-3} i_{n-2, j} \cdot \omega_{j, n}=\sum_{j=1}^{j=n-3} \frac{\overline{K_{n-2} K_{j}}}{\overline{K_{n-2} P_{j}}} \omega_{j, n}
$$

where $P_{j}$ is the intersection of $\bar{r}_{n-1}$ with the displaced vector $\bar{r}_{j}$. Equation (6) is the second relation between the angular velocities of the polygon. Both equations are linear in the angular velocities. In the first equation $\omega_{n-2, n}$ is missing, in the second one $\omega_{n-1, n}$. By choosing another side as the fixed one, we find two other equations which, however, are not independent of the first two. Proceeding in this way we find $2 n$ equations, from which we can keep two for further calculations.

\subsection{The equations for the angular accelerations in the polygon}

Differentiating equation (2) of Euler with respect to time, yields a relation between the acceleration vectors of pivot points $K_{j+1}$ and $\boldsymbol{K}_{j}$

$$
\bar{b}_{\boldsymbol{K}_{j+1}}=\bar{b}_{\boldsymbol{K}_{j}}+\left(\dot{\bar{\omega}}_{j, n} \times \overline{\boldsymbol{r}}_{j}\right)-\omega_{j, n}^{2} \overline{\boldsymbol{r}}_{j}
$$

Summation on $j$ yields

$$
b_{K_{n-2}}=\sum_{j=1}^{j=n-3}\left\{\left(\dot{\bar{\omega}}_{j, n} \times \bar{r}_{j}\right)-\omega_{j, n}^{2} \bar{F}_{j}\right\}=\sum_{j=1}^{j=n-3} b_{K_{n-2}}
$$

On the ground of this equation we can divide the acceleration vector of pivot point $K_{m-2}$ into $n-3$ parts, each of these contributing to the total acceleration vector of pivot point $K_{n-1}$. Each of these contributions can be determined with the help of the four-bar linkage $\left(K_{j} K_{n-2} K_{n-1} K_{n}\right)$, indicated in paragraph 2.1, where ${\vec{K} j K_{n-2}}=\bar{r}_{j}={\vec{K} j K_{j+1}}_{\text {. The angular }}$ acceleration $\dot{\omega}_{n-1, n}$ of side $n-1$ with respect to side $n$ owing to the angular velocity $\omega_{j, n}$ and the angular acceleration $\dot{\omega}_{j, n}$ of the displaced vector $\bar{r}_{j}$, is calculated in the indicated four-bar linkage with the help of an equation, derived by Freudenstein [2]

$$
{ }_{j} \dot{\omega}_{n-1, n}=\lambda_{j} \omega_{j, n}^{2}+i_{n-1, j} \cdot \dot{\omega}_{J, n}
$$

where

$$
\lambda_{j}=i_{n-1, j}\left(1-i_{n-1, j}\right) \operatorname{cotan} \beta_{j}
$$


with $\beta_{j}=\Varangle P_{j}^{\prime} Q_{j}^{\prime} K_{n-1}$

and $\beta_{j}>0$ if the sense of $\Delta P j Q_{j} K_{n-1}$ is counterclockwise and

$\beta_{j}<0$ if the sense of $\Delta P_{j}^{\prime} Q_{j} K_{n-1}$ is clockwise.

Just as $i_{n-1}$, also $\operatorname{cotan} \beta$ can be determined by a division of two linesegments to be measured.

The problem in this case cannot be solved because the actual angular acceleration of link $n-1$ with respect to link $n$ cannot be obtained by simple summation of the separate contributions to the total angular acceleration. So a mixed term has to be added, which we will indicate by $\Delta \dot{\omega}_{n-1, n}$ and which will be calculated afterwards. Then the summation of (9) yields

$$
\dot{\omega}_{n-1, n}-\Delta \dot{\omega}_{n-1, n}=\sum_{j=1}^{j=n-3} j \dot{\omega}_{n-1, n}=\sum_{j=1}^{j=n-3}\left(\lambda, \omega_{j, n}^{2}+i_{n-1, j} . \dot{\omega}_{j, n}\right)
$$

This is one of the equations between the angular accelerations to be formed of the sides. A second equation can be found by choosing another side as the fixed one. Proceeding in this way we find $n$ equations, $n-2$ of them being dependent and 2 being independent.

It can be noticed that, considering link $\boldsymbol{n}$ as fixed, an analogue equation can be formed for $\dot{\omega}_{n-2, n}$. Since this equation will not be used here, we only remark, that this angular acceleration also cannot be calculated by simple addition of the separate contributions. So the mixed term $\Delta \dot{\omega}_{n-2, n}$, defined by the equation

$$
\dot{\omega}_{n-2, n}-\Delta \dot{\omega}_{n-2, n}=\sum_{j=1}^{j=n-3} \dot{\omega}_{n-2, n}
$$

gives the deviation of this addition.

2.2.1 Calculation of the mixed term $\Delta \dot{\omega}_{n-1, n}$. For $j=n-2$ equation (7) turns into

$$
\bar{b}_{K_{n-1}}=\bar{b}_{R_{n-2}}+\left(\dot{\bar{\omega}}_{n-2, n} \times \bar{r}_{n-2}\right)-\omega_{n-2, n}^{2} \bar{n}_{n-2}
$$

Taking a right-handed Cartesian coordinate system with the origin in pivot point $K_{n-1}$, the normal acceleration of which is in the positive direction of the $Y$-axis and the positive tangential acceleration of which is in the positive direction of the $X$-axis with the assumption that the tangential acceleration is called positive if $\dot{\omega}_{n-1, n}$ is counterclockwise, then equation (12) expressed in terms of components gives rise to

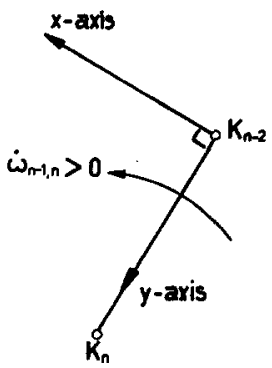

FIG. 2. 


$$
\dot{\omega}_{n-1, n}, r_{n-1}=\left(\bar{b}_{K_{n-2}}\right)_{X}+\left(\dot{\bar{\omega}}_{n-2, n} \times \bar{r}_{n-2}\right)_{X}-\omega_{n-2, n}^{2}\left(\bar{r}_{n-2}\right)_{X}
$$

and

$$
\omega_{n-1, n}^{2} \cdot r_{n-1}=\left(\bar{b}_{K_{n-2}}\right)_{Y}+\left(\dot{\bar{\omega}}_{n-2, n} \times \bar{r}_{n-2}\right)_{Y}-\omega_{n-2, n}^{2}\left(\bar{r}_{n-2}\right)_{Y}
$$

For the $j$ th four-bar linkage $\left(K_{j}^{\prime} K_{n-2} K_{n-1} K_{n}\right)$ we have two similiar equations

$$
{ }_{j} \dot{\omega}_{n-1, n} \cdot r_{n-1}=\left({ }_{j} \bar{b}_{K_{n-2}}\right)_{X}+\left(\dot{\bar{\omega}}_{n-2, n} \times \bar{r}_{n-2}\right)_{X}-{ }_{j} \omega_{n-2, n}^{2}\left(\bar{r}_{n-2}\right)_{X}
$$

and

$$
{ }_{j} \omega_{n-1, n}^{2} \cdot r_{n-1}=\left({ }_{j} \bar{b}_{K_{n-2}}\right)_{Y}+\left({ }_{j} \dot{\bar{\omega}}_{n-2, n} \times \bar{r}_{n-2}\right)_{Y}-{ }_{j} \omega_{n-2, n}^{2}\left(\bar{r}_{n-2}\right)_{Y}
$$

These are two equations valid for each part of the acceleration of the sum

$$
\sum_{j=1}^{j=n-3} j b_{K_{n-2}}=b_{K_{n-2}}
$$

Using the equations (10), (11) and (17), summation of (15) on $j$ yields

$$
\begin{aligned}
\left(\dot{\omega}_{n-1, n}-\Delta \dot{\omega}_{n-1, n}\right) r_{n-1}=\left(\bar{b}_{K_{n-2}}\right)_{X}+\left\{\left(\dot{\bar{\omega}}_{n-2, n}-\Delta \dot{\bar{\omega}}_{n-2, n}\right) \times \bar{r}_{n-2}\right\}_{X} & \\
& -\sum_{j=1}^{j=n-3} \omega_{n-2, n}^{2}\left(\bar{r}_{n-2}\right)_{X} .
\end{aligned}
$$

Taking into account the equations (6) and (13) we have

$$
\Delta \dot{\omega}_{n-1, n} \cdot r_{n-1}=\left(\Delta \dot{\bar{\omega}}_{n-2, n} \times \bar{r}_{n-2}\right)_{X}-\left(\bar{r}_{n-2}\right)_{X} \sum_{\substack{j, l=1 \\ j \neq l}}^{j=n-3} \omega_{n-2, n \cdot l} \omega_{n-2, n}
$$

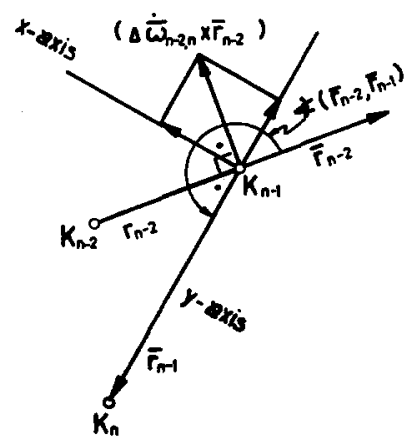

Fro. 3.

or written otherwise

$$
\begin{array}{r}
\Delta \dot{\omega}_{n-1, n} \cdot r_{n-1}=-\Delta \dot{\omega}_{n-2, n} \cdot r_{n-2} \cdot \cos \left(\bar{r}_{n-2}, \bar{r}_{n-1}\right)-r_{n-2} \cdot \sin \left(\bar{r}_{n-2}, \bar{r}_{n-1}\right) \\
\cdot \sum_{\substack{j, l=1 \\
j \neq l}}^{j, l=n-3} \omega_{n-2, n \cdot l} \omega_{n-2, n}
\end{array}
$$


This is an equation between the mixed terms $\Delta \dot{\omega}_{n-1, n}$ and $\Delta \dot{\omega}_{n-2, n}$. Using the equations (11) and (17) summation of (16) on $j$ yields

$$
r_{n-1} \sum_{j=1}^{j=n-3} \omega_{n-1, n}^{2}=\left(\bar{b}_{K_{n-2}}\right)_{Y}+\left\{\left(\dot{\bar{\omega}}_{n-2, n}-\Delta \dot{\bar{\omega}}_{n-2, n}\right) \times \bar{r}_{n-2}\right\}_{Y}-\sum_{j=1}^{j=n-3}{ }_{j} \omega_{n-2, n}^{2}\left(\bar{r}_{n-2}\right)_{Y}
$$

Taking into account the equations (5), (6) and (14) we obtain

$$
r_{n-1} \sum_{\substack{j, l=1 \\ j \neq l}}^{j, l=n-3} \omega_{n-1, n \cdot l} \omega_{n-1, n}=\left(\Delta \dot{\bar{\omega}}_{n-2, n} \times \bar{r}_{n-2}\right)_{Y}-\left(\bar{r}_{n-2}\right)_{Y} \sum_{\substack{j, l=1 \\ j \neq l}}^{j, l=n-3} \omega_{n-2, n \cdot l} \omega_{n-2, n},
$$

so

$$
\begin{aligned}
\Delta \dot{\omega}_{n-2, n}, r_{n-2} & \cdot \sin \left(\bar{r}_{n-2}, \bar{r}_{n-1}\right)=r_{n-1} \sum_{\substack{j, l=1 \\
j \neq l}}^{j, l=n-3} j \omega_{n-1, n \cdot l} \omega_{n-1, n} \\
& +r_{n-2} \cos \left(\bar{r}_{n-2}, \bar{r}_{n-1}\right) \cdot \sum_{\substack{j, j=1 \\
j \neq l}}^{j, l=n-3} \omega_{n-2, n \cdot l} \omega_{n-2, n}
\end{aligned}
$$

With this equation we find the mixed term $\Delta \dot{\omega}_{n-2, n}$. By substituting the result in (18) we get

or

$$
\begin{aligned}
\Delta \dot{\omega}_{n-1, n}=-\{ & \left.\sum_{\substack{j, l=1 \\
j \neq l}}^{J=n-3} \omega_{n-1, n \cdot l} \omega_{n-1, n}+, \omega_{n-2, n} \cdot 1 \omega_{n-2, n} \cdot \frac{r_{n-2}}{r_{n-1}} \cos \left(\bar{r}_{n-2}, \bar{r}_{n-1}\right)\right\} \\
& . \operatorname{cotan}\left(\bar{r}_{n-2}, \bar{r}_{n-1}\right)-\frac{r_{n-2}}{r_{n-1}} \sin \left(\bar{r}_{n-2}, \bar{r}_{n-1}\right) \sum_{\substack{j, l=1 \\
j \neq l}}^{j, l=n-3} \omega_{n-2, n \cdot l} \omega_{n-2, n}
\end{aligned}
$$

$$
\begin{aligned}
\Delta \dot{\omega}_{n-1, n}=- & \sum_{\substack{j, l=1 \\
j \neq l}}^{j, l=n-3} \omega_{n-1, n} \cdot \omega_{n-1, n} \cdot \operatorname{cotan}\left(\bar{r}_{n-2}, \vec{r}_{n-1}\right) \\
& -\frac{r_{n-2}}{r_{n-1} \sin \left(\bar{r}_{n-2}, \bar{r}_{n-1}\right)} \sum_{\substack{j, l=1 \\
j \neq l}}^{j, l=n-3} \omega_{n-2, n \cdot l} \omega_{n-2, n}
\end{aligned}
$$

By using the equations (5) and (6) we obtain finally

$$
\begin{aligned}
\Delta \dot{\omega}_{n-1, n}=- & \sum_{\substack{j, l=1 \\
j \neq l}}^{j \neq l=n-3}\left\{i_{n-1, j}, i_{n-1, l} \cdot \omega_{j, n} \cdot \omega_{l, n} \operatorname{cotan}\left(\bar{r}_{n-2}, \bar{r}_{n-1}\right)\right. \\
& \left.+i_{n-2, j} \cdot i_{n-2, l} \cdot \omega_{j, n} \cdot \omega_{l, n} \frac{r_{n-2}}{r_{n-1} \sin \left(\bar{r}_{n-2}, \bar{r}_{n-1}\right)}\right\}
\end{aligned}
$$

Substituting this result in equation (10) gives an expression for the angular acceleration of the $(n-1)$ th side with respect to the $n$th side 


$$
\begin{gathered}
\dot{\omega}_{n-1, n}=\sum_{j=1}^{j=n-3}\left(\lambda_{j}, \omega_{j, n}^{2}+i_{n-1, j}, \dot{\omega}_{j, n}\right)-\operatorname{cotan}\left(\bar{r}_{n-2}, \bar{r}_{n-1}\right) \sum_{\substack{j, i=1 \\
j \neq l}}^{j, l=n-3} i_{n-1, j}, i_{n-1}, l, \omega_{j, n}, \omega_{l, n} \\
-\frac{r_{n-2}}{r_{n-1} \sin \left(\bar{r}_{n-2}, \bar{r}_{n-1}\right)} \sum_{\substack{j, l=1 \\
j \neq l}}^{j \neq l=n-3} i_{n-2, j}, i_{n-2, l}, \omega_{j, n}, \omega_{l, n}
\end{gathered}
$$

In this equation all coefficients can be taken directly from the diagram and as we presuppose the distribution of the angular velocities to be known, we now have found a useful relation between the angular accelerations of the polygon.

\section{APPLICATION}

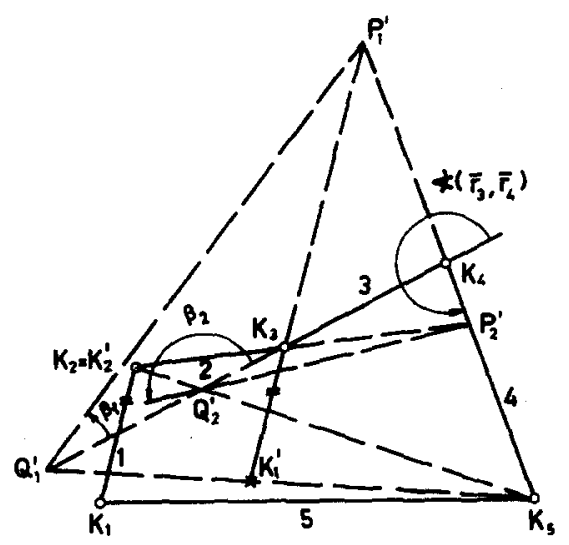

Fio. 4. The pentagon.

As an example we take the pentagon (Fig. 4). With $n=5$ equation (22) gives the relation

$$
\begin{gathered}
\dot{\omega}_{45}=i_{41}\left(1-i_{41}\right) \omega_{15}^{2} \operatorname{cotan} \beta_{1}+i_{42}\left(1-i_{42}\right) \omega_{25}^{2} \operatorname{cotan} \beta_{2}+i_{41} \dot{\omega}_{15}+i_{42} \dot{\omega}_{25} \\
-2 i_{41} i_{42} \omega_{15} \omega_{25} \operatorname{cotan}\left(\bar{r}_{3}, \bar{r}_{4}\right)-2 i_{31} i_{32} \omega_{15} \omega_{25} \frac{r_{3}}{r_{4} \sin \left(\bar{r}_{3}, \bar{r}_{4}\right)} .
\end{gathered}
$$

In this equation we have on the ground of (5)

$$
i_{41}=\frac{\overline{Q_{1} K_{1}^{\prime}}}{\overline{Q_{1} K_{5}}} \text { and } i_{42}=\frac{\overline{Q_{2}^{\prime} K_{2}^{\prime}}}{\overline{Q_{2}^{\prime} K_{5}}}, \quad \text { where } K_{2}^{\prime}=K_{2} \text {, }
$$

and on the ground of (6)

$$
i_{31}=\frac{\overline{K_{3} K_{1}}}{\overline{K_{3} P_{1}^{\prime}}} \text { and } i_{32}=\frac{\overline{K_{3} K_{2}}}{\overline{K_{3} P_{2}^{\prime}}}
$$


The angles $\beta_{1}$ and $\beta_{2}$ together with the angle between the vectors $\bar{r}_{3}$ and $\bar{r}_{4}$ are indicated in the diagram.

For an eight-bar linkage, build up of pentagons only, and having one degree of freedom, this equation has to be applied several times to be able to determine the angular accelerations of each link of the mechanism.

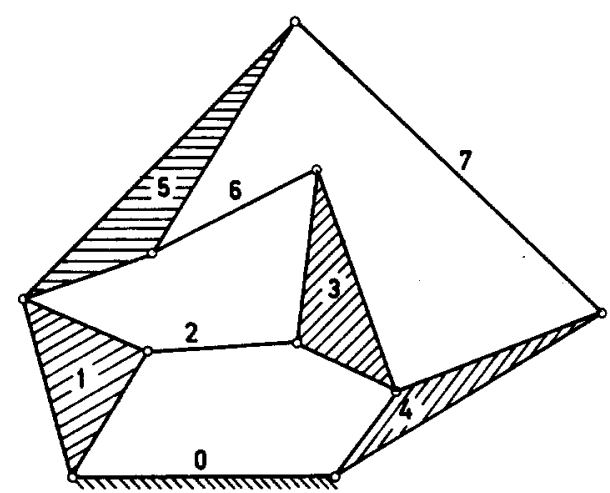

Fig. 5. Mechanism having one degree of freedom and build up of only pentagons.

A mechanism with $n$ links has in general $\frac{1}{2} n(n-1)$ angular accelerations of the links with respect to each other if $\dot{\omega}_{i k}$ and $\dot{\omega}_{k i}$ (with the connection $\dot{\omega}_{i k}=-\dot{\omega}_{k i}$ ) are not counted separately. Where $n=8$ in the mechanism as presented in Fig. 5, there are so $\frac{1}{2} \times 8.7=28$ different angular accelerations present on a fixed moment. Between those we have $\frac{1}{2}(n-1)$ $(n-2)=\frac{1}{2} \times 7.6=21$ independent relations of the kind $\dot{\omega}_{i j}+\dot{\omega}_{j k}=\dot{\omega}_{i k}$. (The proof of which will be delivered at the end of this paragraph.) Where the angular acceleration of the input link with respect to the fixed one is given, there are so $28-1-21=6$ independent relations bound to be found to solve the problem. As we have seen, it is possible to extract from each pentagon two independent graphical relations. So three different pentagons specifiable in the mechanism are quite sufficient to find all angular accelerations. For example one can take the pentagons $1-2-3-4-0,1-5-7-4-0$ and $1-5-6-3-2$.

In the case one only wants to know the angular acceleration of the output link with respect to the fixed link, a lesser number of relations is sufficient to solve the problem.

If there are $n$ links, the number of independent relations of the kind $\dot{\omega}_{i j}+\dot{\omega}_{j k}=\dot{\omega}_{i k}$ can be determined as follows:

Let this number be $T$. For a $n$-side polygon there are $(n-3)$ angular accelerations to choose which are independent of each other. There remain some $\left\{\frac{1}{2} n(n-1)-(n-3)\right\}$ angular accelerations which are to be calculated from a same number of independent relations. The latter are the two graphical relations, already mentioned, and the $T$ independent relations of the kind $\dot{\omega}_{i j}+\dot{\omega}_{j k}=\dot{\omega}_{i k}$ always present between $n$ moving bodies. Hence:

Thus

$$
\frac{1}{2} n(n-1)-(n-3)=2+T \text {. }
$$

$$
T=\frac{1}{2}(n-1)(n-2), *
$$

* A relation which was to be proved. 


\section{SUMMARY}

For a mechanism of bars, the links being connected by turning pairs only and having one degree of freedom, an accurate graphic method has been developed to determine the angular acceleration of the output link. It is based on the consideration, that for the determination of the movement of a link with number $n-1$ with respect to the jointed link $n$, the $n$-side polygon, to which the two links belong, can be split up into $(n-3)$ four-bar linkages, each having 2 sides coinciding with the sides $(n-2)$ and $(n-1)$ of the $n$-side polygon. A third side is equal and parallel in turn to one of the $(n-3)$ sides preceding to the $(n-2)$ th side. The fourth side, which is the closing side, is considered kinematically the same as link $n$. Each of the $(n-3)$ four-bar linkages contributes to the angular acceleration of link $(n-1)$ with respect to link $n$ of the polygon. An additional contribution represented by a mixed term, has to be taken into account. This term is calculated in a separate paragraph of this paper.

By cyclic permutation of the link $n$ of the polygon which is considered to be fixed, we can find $2 n$ relations between the angular accelerations of the $n$ sides. $(2 n-2)$ of these equations are dependent while 2 are independent. It is always possible to find the minimum number of equations necessary to determine the angular acceleration of the output link by right choice of the needed polygons of the mechanism and of the side considered to be fixed in each of them. The method is exceedingly fit for extension to mechanisms with more than one degree of freedom. For mechanisms with rotatable sliding pairs as well as turning pairs the method can also be extended.

\section{REFERENCES}

[1] N. Rosenauer, On the Construction of Accelerations of Kinematic Chains and Mechanisms. Contributions Baltic University, no. 32 Pinneberg (1947).

[2] F. Freudenstein, On the maximum and minimum velocities and the accelerations in four-link mechanisms. ASME-Paper, Nr. 55-SA-20. 